PROCEEDINGS OF THE

AMERICAN MATHEMATICAL SOCIETY

Volume 133, Number 11, Pages 3285-3293

S 0002-9939(05)07839-1

Article electronically published on May 2, 2005

\title{
ON COMMUTING OPERATORS SOLVING GLEASON'S PROBLEM
}

\author{
D. ALPAY AND C. DUBI
}

(Communicated by Joseph A. Ball)

\begin{abstract}
We prove the uniqueness of commuting operators solving Gleason's problem for certain spaces of functions analytic in the unit ball.
\end{abstract}

\section{INTRODUCTION}

The backward shift operator at a point $a \in \mathbb{C}$ is defined by

$$
R_{a} f=\frac{f(z)-z(a)}{z-a} .
$$

The backward shift operator and the study of backward shift invariant spaces play an important role in one complex variable and appear in a variety of topics such as operator models, linear system theory, realization theory of matrix-valued rational functions and more. When going to the setting of several complex variables, equation (1.1) no longer makes sense, and the question of backward shift invariance can be replaced with the following problem:

Problem 1.1. Let $\mathcal{X}$ be a set of functions analytic in $\Omega \subset \mathbb{C}^{N}$. Given $f \in \mathcal{X}$ and $a \in \Omega$, do functions $g_{1}, g_{2}, \ldots, g_{n} \in \mathcal{X}$ exist such that

$$
f(z)-f(a)=\sum_{j=1}^{N}\left(z_{j}-a_{j}\right) g_{j}(z)
$$

for every $z \in \Omega$ ?

This is Gleason's problem, originally set for $a=0$ and in the setting of functions analytic in the unit ball $\mathbb{B}_{N}$ of $\mathbb{C}^{N}$ and continuous on the boundary of the unit ball. For $N=1$, solving Gleason's problem is just to establish whether or not $\mathcal{X}$ is invariant under the backward shift operator $R_{a}$.

Gleason's problem can be studied through different points of view. The first one asks only whether or not the requested functions $g_{1}, g_{2}, \ldots, g_{N} \in \mathcal{X}$ exist. A second point of view is operator theoretical: one assumes that the multiplication operator $M_{z_{j}}: f(z) \mapsto z_{j} f(z)$ is continuous on $\mathcal{X}$ for each $j=1, \ldots, N$ and then studies the existence and properties of $N$ bounded operators $T_{a, j}: \mathcal{X} \rightarrow \mathcal{X}$ such that for every

Received by the editors February 3, 2004 and, in revised form, June 12, 2004.

2000 Mathematics Subject Classification. Primary 47B32.

(C)2005 American Mathematical Society 
$f \in \mathcal{X}$

$$
f(z)-f(a)=\sum_{j=1}^{N}\left(z_{j}-a_{j}\right) T_{a, j} f(z) .
$$

We will say that a set of operators $\left\{T_{a, 1}, T_{a, 2}, \ldots, T_{a, N}\right\}$ solves Gleason's problem (with center point $a$ ) if equality (1.3) is satisfied.

Again, when restricting to $N=1$, there can be only one operator solving Gleason's problem, namely the backward shift operator $R_{a}$. When turning to $N>1$, this is obviously not the case; as will be demonstrated in the next example, even in simple cases the operators solving Gleason's problem are not uniquely defined.

Example 1.2. Set $\mathcal{M}$ to be the finite-dimensional space of functions on $\mathbb{C}^{2}$ spanned by the functions

$$
f_{1}=1, f_{2}=z_{1}, f_{3}=z_{2}, f_{4}=z_{1} z_{2} .
$$

It is easy to check that the operators $A_{1}, A_{2}$ defined by

$$
\begin{array}{r}
A_{1} f_{1}=0, A_{1} f_{2}=1, A_{1} f_{3}=0, A_{1} f_{4}=z_{2}, \\
A_{2} f_{1}=0, A_{2} f_{2}=0, A_{2} f_{3}=1, A_{2} f_{4}=0
\end{array}
$$

solve Gleason's problem in $\mathcal{M}$. On the other hand, taking $B_{1}, B_{2}$ defined by

$$
\begin{aligned}
& B_{1} f_{1}=0, B_{1} f_{2}=1, B_{1} f_{3}=0, B_{1} f_{4}=0, \\
& B_{2} f_{1}=0, B_{2} f_{2}=0, B_{2} f_{3}=1, B_{2} f_{4}=z_{1}
\end{aligned}
$$

we have another set of operators solving Gleason's problem.

The main result of the present work asserts that the non-uniqueness vanishes when the operators $\left\{T_{1}, T_{2}, \ldots, T_{N}\right\}$ are pairwise commuting; see Theorem 4.1 in section 4

We conclude this introduction with some notation and definitions that will be used in the sequel. For $\alpha=\left(\alpha_{1}, \alpha_{2}, \ldots, \alpha_{N}\right) \in \mathbb{N}^{N}$, we denote

$$
|\alpha|=\sum_{j=1}^{N} \alpha_{j}, \quad \alpha !=\prod_{j=1}^{N} \alpha_{j} !
$$

If $T=\left(T_{1}, T_{2}, \ldots, T_{N}\right)$ is a set of $N$ commuting, bounded operators, we set

$$
T^{\alpha}=\prod_{j=1}^{N} T_{j}^{\alpha_{j}} .
$$

Similarly, if $z \in \mathbb{C}^{N}$, we set $z^{\alpha}=\prod_{j=1}^{N} z_{j}^{\alpha_{j}}$.

Definition 1.3. Let $T=\left(T_{1}, T_{2}, \ldots, T_{N}\right)$ be a set of $N$ commuting, bounded operators on a Hilbert space. We denote by $\Delta(T)$ the largest domain containing the origin for which the operator

$$
\sum_{\alpha \in \mathbb{N}^{N}} \frac{|\alpha| !}{\alpha !} w^{\alpha} T^{\alpha}
$$

converges when $w \in \Delta(T)$. 
Since we assume that $\left(T_{1}, T_{2}, \ldots, T_{N}\right)$ are all bounded, it is clear that $\Delta(T)$ is an open set containing the origin. Moreover, since $\left(T_{1}, T_{2}, \ldots, T_{N}\right)$ commute, one has that

$$
\left(I-\sum_{j=1}^{N} z_{j} T_{j}\right)^{-1}=\sum_{\alpha \in \mathbb{N}^{N}} \frac{|\alpha| !}{\alpha !} z^{\alpha} T^{\alpha}
$$

for every $w \in \Delta(T)$, and thus the operator $\left(I-\sum_{j=1}^{N} w_{j} T_{j}\right)^{-1}$ is well defined for $w=\left(w_{1}, \ldots, w_{N}\right)$ for $w \in \Delta(T)$.

\section{SPACES OF ANALYTIC FUnCTIONS AND WEIGHTED POWER SERIES SPACES}

Let $\mathcal{P}$ be a reproducing kernel Hilbert space of $\mathbb{C}^{p}$-valued functions analytic on a connected open subset $\Omega \subset \mathbb{C}^{N}$ for which the operator of multiplication by the $j$-th coordinate $z_{j}$

$$
M_{z_{j}}: f(z) \mapsto z_{j} f(z),
$$

is continuous on $\mathcal{P}$. By the closed graph theorem, it suffices to assume that the space $\mathcal{P}$ is invariant under $M_{z_{j}}$ for each $j$. The reproducing-kernel assumption means that, for each $a \in \Omega$, the evaluation mapping $E(a): \mathcal{P} \mapsto \mathbb{C}^{p}$ given by $f \mapsto f(a)$ is continuous. A standard fact then is that the map $f \mapsto \xi_{\alpha} \in \mathbb{C}^{p}$ is continuous where $\xi_{\alpha}$ is the $\alpha$-coefficient in the power series expansion for $f$ at $a$ :

$$
f(z)=\sum_{\alpha \in \mathbb{N}^{N}} \xi_{\alpha} z^{\alpha}
$$

Particular examples of such reproducing-kernel Hilbert spaces are the spaces with the following structure: we are given a weight sequence $\left\{a_{\alpha}\right\}_{\alpha \in \mathbb{N}^{N}}$ consisting of nonnegative real numbers $a_{\alpha} \geq 0$ and then define a spaces $\mathcal{P}$ by

$$
\mathcal{P}=\left\{\sum_{\alpha \in \mathbb{N}^{N}} z^{\alpha} \xi_{\alpha} \mid \quad \xi_{\alpha} \in \mathbb{C}^{p}, \sum_{\alpha \in \mathbb{N}^{N}} a_{\alpha}\left\|\xi_{\alpha}\right\|_{p}^{2}<\infty\right\}
$$

where $\|\cdot\|_{p}$ is the standard norm of $\mathbb{C}^{p}$. We refer to such spaces $\mathcal{P}$ as the weighted power series space associated with the weight sequence $\left\{a_{\alpha}\right\}_{\alpha \in \mathbb{N}^{N}}$. We notice that $\mathcal{P}$ is a Hilbert space of $\mathbb{C}^{p}$-valued functions, where the inner product in $\mathcal{P}$ is given by

$$
\langle f, g\rangle_{\mathcal{P}}=\sum_{\alpha \in \mathbb{N}^{N}} a_{\alpha} g_{\alpha}^{*} f_{\alpha}
$$

where $f(z)=\sum_{\alpha \in \mathbb{N}^{N}} f_{\alpha} z^{\alpha}$ and $g(z)=\sum_{\alpha} g_{\alpha} z^{\alpha}$. In the literature, together with the above-mentioned inner product, $\mathcal{P}$ is sometimes called a weighted Hardy space. However, the fact that $\left\langle z^{\alpha}, z^{\beta}\right\rangle=0$ for $\alpha \neq \beta$ has no importance in the proof, and we will drop this assumption on the inner product of $\mathcal{P}$ (in such a case, $\mathcal{P}$ is no longer a weighted Hardy space).

Let $\mathcal{P}$ be a reproducing kernel Hilbert space of $\mathbb{C}^{p}$-valued functions over the domain $\Omega \subset \mathbb{C}^{N}$ as above and let $a$ be a point of $\Omega$. We now define

$$
L_{\mathcal{P}}^{a}=\left\{\left\{\xi_{\alpha}\right\}_{\alpha \in \mathbb{N}^{N}} \mid \quad \xi_{\alpha} \in \mathbb{C}^{p}, \quad \sum_{\alpha \in \mathbb{N}^{N}}(z-a)^{\alpha} \xi_{\alpha} \in \mathcal{P}\right\}
$$


and the isomorphism $\Phi^{a}: L_{\mathcal{P}}^{a} \rightarrow \mathcal{P}$ in the following manner: for every $\xi=$ $\left\{\xi_{\alpha}\right\}_{\alpha \in \mathbb{N}^{N}}$, the action of $\Phi$ on $\left\{\xi_{\alpha}\right\}_{\alpha \in \mathbb{N}^{N}}$ is

$$
\left(\Phi^{a} \xi\right)(z)=\sum_{\alpha \in \mathbb{N}^{N}}(z-a)^{\alpha} \xi_{\alpha} .
$$

$L_{\mathcal{P}}^{a}$ is the "time-domain version" of $\mathcal{P}$ (using the center point $a \in \Omega$ ), and the mapping $\Phi$ amounts to the $N$-variable $Z$-transform. As $\Omega$ is connected, by the principle of analytic continuation we see that any element $f \in \mathbb{P}$ is uniquely determined by its sequence of Taylor coefficients $\left\{\xi_{\alpha}\right\}$ associated with the center point $a$. We view $L^{a}(\mathcal{P})$ as a Hilbert space with norm inherited from $\mathcal{P}$ :

$$
\|\xi\|_{L_{\mathcal{P}}^{a}}=\left\|\Phi^{a}(\xi)\right\|_{\mathcal{P}} .
$$

Next we define the operator $F^{a}(z): \Omega \rightarrow \mathcal{L}\left(L_{\mathcal{P}}^{a}, \mathbb{C}^{q}\right)$ as $F^{a}(z)=E(z) \circ \Phi^{a}$, i.e.,

$$
F^{a}(z) \xi=\sum_{\alpha \in \mathbb{N}^{N}}(z-a)^{\alpha} \xi_{\alpha} .
$$

For each fixed $z=\left(z_{1}, \ldots, z_{N}\right)$, by linearity we have

$$
z_{j} F(z) \xi=F(z)\left(z_{j} \xi\right) .
$$

At the level of operators we have

$$
M_{z_{j}-a_{j}} \Phi \xi=\Phi S_{j} \xi
$$

where $S_{j}$ is the shift operator given by

$$
S_{j}:\left\{\xi_{\alpha}\right\}_{\alpha \in \mathbb{N}^{N}} \mapsto\left\{\eta_{\alpha}\right\}_{\alpha \in \mathbb{N}^{N}}
$$

where $\eta_{\alpha}=0$ if $\alpha_{j}=0$ and $\eta_{\alpha}=\xi_{\alpha-e_{j}}$ otherwise.

\section{The Ahern-Schneider And Leibenson solutions}

\section{TO GLEASON'S PROBLEM}

In [9], Gleason's problem was discussed in the setting of the Hardy space of the ball, and the following formula was given: for $f \in \mathcal{P}$, define

$$
R_{j, a} f(z)=\int_{\mathbb{S}_{N}} \frac{C(z, w)-C(a, w)}{\langle z-a, w\rangle} \bar{w}_{j} f(w) d \sigma(w),
$$

where $d \sigma$ denotes the Lebesgue measure on the unit sphere $\mathbb{S}_{N}$ in $\mathbb{C}^{N}$ and $C(z, w)=$ $(1-\langle z, w\rangle)^{-N}$ is the Cauchy kernel of $\mathbb{B}_{N}$. Then

$$
f(z)-f(a)=\sum_{j}^{N}\left(z_{j}-a_{j}\right) R_{j, a} f(z) .
$$

Formula (3.5) is due to Ahern-Schneider.

One easily checks that the set $R=\left\{R_{1, a}, R_{2, a}, \ldots, R_{N, a}\right\}$ is pairwise commuting. For the case $a=0$, formula (3.5) can be seen to be equal to

$$
\left(R_{j} f\right)(z)=\int_{0}^{1} \frac{\partial f}{\partial z_{j}}(t z) d t .
$$

The Leibenson formula (3.6) can also be described by its action on the monomials.

One can check that

$$
\left(R_{j} z^{\alpha}\right)=\left\{\begin{array}{l}
\frac{\alpha_{j}}{|\alpha|} z^{\alpha-e_{j}} ; \quad \alpha_{j} \geq 1, \\
0 ; \quad \alpha_{j}=0
\end{array}\right.
$$


and thus the time-domain version of $R_{j}$ is given by $\left\{\xi_{\alpha}\right\} \mapsto\left\{\eta_{\alpha}\right\}$ where

$$
\eta_{\alpha}=\frac{\left(\alpha+e_{j}\right)_{j}}{\left|\alpha+e_{j}\right|} \xi_{\alpha+e_{j}}=\frac{\alpha_{j}+1}{|\alpha|+1} \xi_{\alpha+e_{j}} .
$$

These operators were defined by Leibenson; see [9] Section 6.6]. The Leibenson solution has been studied by the authors in [2, where it was introduced as the generalization of the backward shift operator for functions in $N$ variables. In 5 E. Doubtsov proved that the Leibenson solution is a minimal solution (in an appropriate sense) to Gleason's problem at $a=0$. He also used the Leibenson solution to study composition operators in the ball; see [6].

We will see in the sequel that the Ahern-Schneider solution is the only commuting solution to Gleason's problem in the general setting of reproducing-kernel Hilbert spaces invariant under multiplication by the coordinate functions.

\section{MAIN THEOREM}

Theorem 4.1. Let $\mathcal{P}$ be a space of $\mathbb{C}^{p}$-valued functions analytic on a domain $\Omega \subset \mathbb{C}^{N}$ which is invariant under the multiplication operators $M_{z_{j}}$ for $j=1, \ldots, N$, and let $a \in \Omega$. The set of commuting, bounded operators solving Gleason's problem with center point a in $\mathcal{P}$, if it exists, is unique, and is given by

$$
T_{j}: f(z) \mapsto g_{j}(z)
$$

where $g_{j}(z)$ is the uniquely determined element of $\mathcal{P}$ having Taylor expansions with center point a given by

$$
g_{j}(z)=\sum_{\alpha \in \mathbb{N}^{N}: \alpha_{j} \geq 1}(z-a)^{\alpha-e_{j}} \frac{\alpha_{j}}{|\alpha|} \xi_{\alpha}
$$

if $f(z)$ has Taylor expansion at a given by

$$
f(z)=\sum_{\alpha \in \mathbb{N}^{N}}(z-a)^{\alpha} \xi_{\alpha} .
$$

Proof. To simplify the notation we assume that $0 \in \Omega$ and that $a=0$. We then write simply $\Phi$ in place of $\Phi^{0}$ and $F(z)$ in place of $F^{0}(z), L_{\mathcal{P}}$ in place of $L_{\mathcal{P}}^{0}$, etc. Assume that $T=\left\{T_{1}, T_{2}, \ldots, T_{N}\right\}$ is a set of commuting, bounded operators solving Gleason's problem in $\mathcal{P}$ at 0 . Denote by $A=\left\{A_{1}, A_{2}, \ldots, A_{N}\right\}, A_{j}: L_{\mathcal{P}} \rightarrow L_{\mathcal{P}}$ the time-domain version of $T$ on $L_{\mathcal{P}}$, that is,

$$
A_{j} \xi=\eta \quad \text { if and only if } \quad T_{j}(\Phi \xi)=\Phi \eta \text {. }
$$

The operators $A_{j}$ are given by the formula $A_{j}=\Phi^{-1} T_{j} \Phi$. Clearly, $A_{i} A_{j}=A_{j} A_{i}$ for every $i, j$. By the definition of the norm on $L_{\mathcal{P}}$, the operators $A_{j}$ on $L_{\mathcal{P}}$ are unitarily equivalent to $T_{j}$ on $\mathcal{P}$, and hence are bounded. Next, since $T$ solves Gleason's problem, it follows that

$$
F(z) \xi-F(0) \xi=\sum_{j=1}^{N} z_{j} F(z) A_{j} \xi
$$

and hence also

$$
F(z)\left(I-\sum_{j=1}^{N} z_{j} A_{j}\right)=F(0)
$$


for every $\xi \in L_{\mathcal{P}}$ for all $z \in \Omega$. Using the assumption that $T_{1}, \ldots, T_{N}$ commute, by (1.4) we get, for every $z \in \Delta(A)$,

$$
F(z)=F(0)\left(I-\sum_{j=1}^{N} z_{j} A_{j}\right)^{-1}=\sum_{\alpha \in \mathbb{N}^{N}} \frac{|\alpha| !}{\alpha !} z^{\alpha} F(0) A^{\alpha} .
$$

Thus, for $\xi \in L_{\mathcal{P}}$,

$$
\xi_{\alpha}=\frac{|\alpha| !}{\alpha !} F(0) A^{\alpha} \xi
$$

On the other hand, for every $z \in \Delta(A)$ and $\xi \in L_{\mathcal{P}}$,

$$
\begin{aligned}
\left(T_{j} \Phi \xi\right)(z) & =\left(\Phi A_{j} \xi\right)(z)=F(z) A_{j} \xi=\sum_{\alpha \in \mathbb{N}^{N}} \frac{|\alpha| !}{\alpha !} z^{\alpha} F(0) A^{\alpha} A_{j} \xi \\
& =\sum_{\substack{\alpha \in \mathbb{N}^{N} \\
\alpha_{j} \geq 1}} z^{\alpha-e_{j}} \frac{\left|\alpha-e_{j}\right| !}{\left(\alpha-e_{j}\right) !} F(0) A^{\alpha} \xi \\
& =\sum_{\substack{\alpha \in \mathbb{N}^{N} \\
\alpha_{j} \geq 1}} z^{\alpha-e_{j}} \frac{\alpha_{j}}{|\alpha|} \frac{|\alpha| !}{\alpha !} F(0) A^{\alpha} \xi \\
& =\sum_{\substack{\alpha \in \mathbb{N}^{N} \\
\alpha_{j} \geq 1}} z^{\alpha-e_{j}} \xi_{\alpha} \frac{\alpha_{j}}{|\alpha|},
\end{aligned}
$$

and it follows that $T_{j}$ is given by (4.9) as asserted.

\section{EXAMPLES AND APPLICATIONS}

In this section we study some consequences of the main theorem. The first is the following characterization:

Theorem 5.1. Let $\mathcal{H}$ be a weighted power series space associated with the sequence $\left\{\phi_{\alpha}\right\}_{\alpha \in \mathbb{N}^{N}}$. Then $\mathcal{H}$ has a commuting set of operators solving Gleason's problem at 0 if and only if

$$
\sup _{\alpha \in \mathbb{N}^{N}} \frac{\alpha_{j}}{|\alpha|} \frac{\phi_{\alpha-e_{j}}}{\phi_{\alpha}}<\infty \quad \text { for } \quad i=1,2, \ldots, N .
$$

Proof. Assume that condition (5.11) holds. Then $\left\{R_{j}\right\}_{j=1}^{N}$ as defined in (3.6) are bounded on $\mathcal{H}$. Clearly $\left\{R_{j}\right\}_{j=1}^{N}$ form a commuting set of operators solving Gleason's problem at 0 , as stated.

On the other hand, if a commuting set of bounded operators $\left\{T_{j}\right\}_{j=1}^{N}$ solves Gleason's problem, through Theorem 4.1 it is evident that $T_{j}=R_{j}$. Since

$$
\frac{\left\|R_{j} z^{\alpha}\right\|^{2}}{\left\|z^{\alpha}\right\|^{2}}=\frac{\alpha_{j}}{|\alpha|} \frac{\phi_{\alpha-e_{j}}}{\phi_{\alpha}}
$$

this last expression is bounded for every $\alpha \in \mathbb{N}^{N}$, and thus the statement.

Example 5.2. Let $\phi_{\alpha}=\frac{1}{(\alpha !)}$ and let $\mathcal{H}$ be the weighted power series space associated to $\left\{\phi_{\alpha}\right\}_{\alpha \in \mathbb{N}^{N}}$. Since

$$
\frac{\alpha_{j}}{|\alpha|} \frac{\phi_{\alpha-e_{j}}}{\phi_{\alpha}}=\frac{\alpha_{j}}{|\alpha|} \frac{\alpha !}{\left(\alpha-e_{j}\right) !}=\frac{\alpha_{j}^{2}}{|\alpha|}
$$


taking $\alpha=n e_{j}$, we have

$$
\frac{\alpha_{j}}{|\alpha|} \frac{\phi_{\alpha-e_{j}}}{\phi_{\alpha}}=n
$$

Thus there is no set of commuting bounded operators on $\mathcal{H}$ solving Gleason's problem at 0 .

For the next example, we recall that the Arveson space is the reproducing-kernel Hilbert space $\mathcal{A}$ of functions analytic in the unit ball of $\mathbb{C}^{N}$ and with reproducing kernel

$$
K(z, w)=\frac{1}{1-\langle z, w\rangle} \quad \text { where } \quad\langle z, w\rangle=\sum_{1}^{N} z_{j} \overline{w_{j}} .
$$

It is the weighted power series space associated to the sequence $\phi_{\alpha}=\frac{\alpha !}{|\alpha| !}$. It is strictly included in the Hardy space of the ball.

Theorem 5.3. Denote by $T_{j, a}$ the operator of multiplication by $\frac{z_{j}}{1-\langle z, a\rangle}$ in $\mathcal{A}$. Then

$$
T_{j, a}^{*} f=R_{j, a} f=\int_{\mathbb{S}_{N}} \frac{C(z, w)-C(a, w)}{\langle z-a, w\rangle} \overline{w_{j}} f(w) d \sigma(w)
$$

where $*$ denotes the adjoint.

Proof. We first recall that $R_{j, a}$ is well defined on the Hardy space, and thus defined on $\mathcal{A}$ (still, it is not clear that $R_{j, a}$ maps $\mathcal{A}$ into itself). It was proved in [3] that the operators $\left\{T_{j, a}^{*}\right\}$ are bounded and form a commuting solution to Gleason's problem in $\mathcal{A}$. Hence, by Theorem 4.1, both $T_{j, a}^{*}$ and $R_{j, a}$ satisfy

$$
T_{j, a}(z-a)^{\alpha}=R_{j, a}(z-a)^{\alpha}=\frac{\alpha_{j}}{|\alpha|}(z-a)^{\alpha-e_{j}} .
$$

Since the polynomials form a dense orthogonal set in $\mathcal{A}$, this implies that $R_{j, a}$ is bounded in $\mathcal{A}$, and thus maps $\mathcal{A}$ into itself. Applying Theorem 4.1 will then prove the theorem.

Remark 5.4. For $N=2$, the result of Theorem 5.3 was proved by direct computations in [3]. We also remark that in view of Theorem [5.3, we have the evaluation

$$
\int_{\mathbb{S}_{N}} \frac{C(z, w)-C(a, w)}{\langle z-a, w\rangle} \overline{w_{j}}(w-a)^{\alpha} d \sigma(w)=\frac{\alpha_{j}}{|\alpha|}(z-a)^{\alpha-e_{j}} .
$$

Similarly, in case $a=0$, we recover the formula 3.7 -3.8 for the Leibenson shifts (3.6) independently as a consequence of Theorem 4.1 combined with the fact that they solve the Gleason problem at $a=0$.

In [4], another method of solution to Gleason's problem was described in the space $\mathcal{A}$ and in other reproducing-kernel Hilbert spaces whose kernels are analytic functions of $\langle z, w\rangle$, such as Dirichlet-type spaces.

In the next theorem we study the relationship between commuting solutions to Gleason's problem at different points of $\mathbb{B}_{N}$.

Theorem 5.5. Let $R_{a}$ and $R_{b}$ be two sets of commuting operators solving Gleason's problem at $a$ and $b$ respectively in a Hilbert space $\mathcal{P}$ of functions analytic in some domain of $\mathbb{C}^{N}$. If $(b-a) \in \Delta\left(R_{a}\right)$, then

$$
R_{j, b}=R_{j, a}\left(I-\sum_{j=1}^{N}\left(b_{j}-a_{j}\right) R_{a, j}\right)^{-1} \text {. }
$$


Proof. In view of Theorem 4.1, it is sufficient to show that

$$
\left\{R_{j, a}\left(I-\sum_{j=1}^{N}\left(b_{j}-a_{j}\right) R_{a, j}\right)^{-1}\right\}_{j=1}^{N}
$$

are a commuting set of operators solving Gleason's problem at $b$. The fact that these operators commute is trivial. We thus turn to prove that they solve Gleason's problem at the point $b$. As in Theorem 4.1, we can write

$$
F(z)=F(a)\left(I-\sum_{j}\left(z_{j}-a_{j}\right) R_{a, j}\right)^{-1} .
$$

Since $(b-a) \in \Delta\left(R_{a}\right)$, the last holds for $z=b$. Thus for $\xi \in L_{\mathcal{P}}$ we have

$$
\begin{aligned}
F(z) \xi-F(b) \xi= & F(a)\left(I-\sum_{j}\left(z_{j}-a_{j}\right) R_{a, j}\right)^{-1} \xi-F(a)\left(I-\sum_{j}\left(b_{j}-a_{j}\right) R_{a, j}\right)^{-1} \xi \\
= & F(a)\left(I-\sum_{j}\left(z_{j}-a_{j}\right) R_{a, j}\right)^{-1}\left(\sum_{j}\left(z_{j}-b_{j}\right) R_{a, j}\right) \\
& \times\left(I-\sum_{j}\left(b_{j}-a_{j}\right) R_{a, j}\right)^{-1} \xi \\
= & \sum_{j}\left(z_{j}-b_{j}\right) F(z) R_{a, j}\left(I-\sum_{j}\left(b_{j}-a_{j}\right) R_{a, j}\right)^{-1} \xi,
\end{aligned}
$$

and the theorem is proved.

\section{ACKNOWLEDGMENT}

It is our pleasure to thank the referee for his careful reading of this paper and his valuable remarks.

\section{REFERENCES}

[1] D. Alpay. The Schur algorithm, reproducing kernel spaces and system theory. American Mathematical Society, Providence, RI, 2001. Translated from the 1998 French original by Stephen S. Wilson, Panoramas et Synthèses. [Panoramas and Syntheses]. MR.1839648 (2002b:47144)

[2] D. Alpay and C. Dubi. Backward shift operator and finite dimensional de Branges Rovnyak spaces in the ball. Linear Algebra Appl., 371:277-285, 2003. MR.1997376 (2004e:46035)

[3] D. Alpay and H.T. Kaptanoğlu. Some finite-dimensional backward-shift-invariant subspaces in the ball and a related interpolation problem. Integral Equation Operator Theory, 42:1-21, 2002. MR 1866874 (2002i:47007)

[4] D. Alpay and H.T. Kaptanoğlu. Gleason's problem and homogeneous interpolation in Hardy and Dirichlet-type spaces of the ball. J. Math. Anal. Appl., 276:654-672, 2002. MR1944782 (2004f:46040)

[5] E. Doubtsov. Minimal solutions of the Gleason problem. Complex Var. Theory Appl., 36(1):27-35, 1998. MR1637332 (99i:32007)

[6] E. Doubtsov. Leibenzon's backward shift and composition operators. Proc. Amer. Math. Soc., 129(12):3495-3499 (electronic), 2001. MR.1860481 (2002j:47046)

[7] H. Dym. J-contractive matrix functions, reproducing kernel Hilbert spaces and interpolation. Published for the Conference Board of the Mathematical Sciences, Washington, DC, 1989. MR.1004239 (90g:47003)

[8] A. Gleason. Finitely generated ideals in Banach algebras. J. Math. Mech., 13:125-132, 1964. MR0159241 (28:2458) 
[9] W. Rudin. Function theory in the unit ball of $\mathbb{C}^{n}$. Springer-Verlag, 1980. MR0601594 (82i:32002)

[10] E.L. Stout. The theory of uniform algebras. Bogden \& Quigley, Inc., Tarrytown-on-Hudson, N. Y., 1971. MR0423083 (54:11066)

Department of Mathematics, Ben-Gurion University of the Negev, Beer-Sheva 84105, ISRAEL

E-mail address: dany@math.bgu.ac.il

Department of Mathematics, Ben-Gurion University of the Negev, Beer-Sheva 84105, ISRAEL

E-mail address: dubi@math.bgu.ac.il

Current address: Department of Mathematics, Weizmann Institute, POB 26, Rehovot 76100, Israel

E-mail address: dubic@weizmann.ac.il 